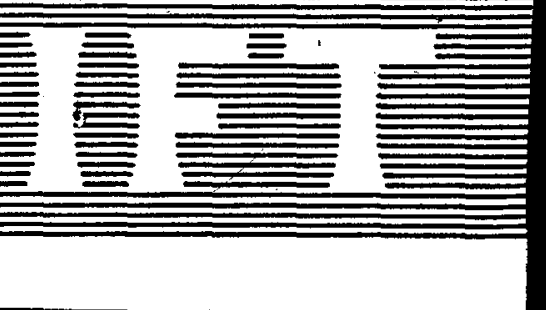

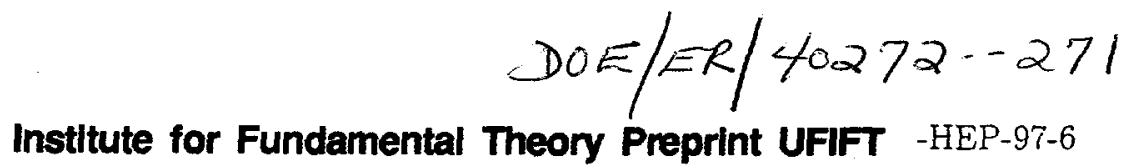

March 1997

\title{
RELATIVISTIC CHARGED PARTICLE IN THE \\ DIPOLE-SPHERE CONFIGURATION II. GENERAL TILTED \\ SURFACE ORBITS
}

\section{Kaundinya S. Gopinath* \\ Dallas C. Kennedy ${ }^{\dagger}$}

Department of Physics, University of Florida, Gainesville, Florida 32611 USA
PECPIMES

APR 071997
OSFI

\begin{abstract}
Relativistic charged particle orbits on a rotating sphere threaded by an intense magnetic dipole field are examined. Generalizing the results of the first paper (I), the dipole and rotational axes have arbitrary relative tilt. For ultra-intense magnetic fields characteristic of compact astrophysical bodies, the classical and semiclassical results are not greatly changed for moderate rotation rates.

PACS numbers: $03.65 . \mathrm{Sq}, 95.85 . \mathrm{Nv}, 97.60 . \mathrm{Gb}, 97.60 . \mathrm{Jd}$
\end{abstract}

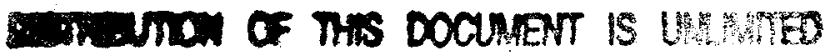
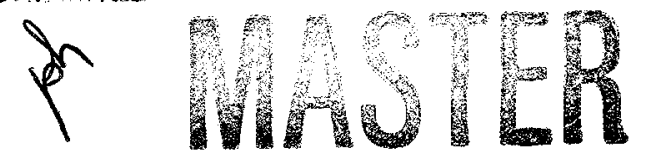


\section{DISCLAIMER}

This report was prepared as an account of work sponsored by an agency of the United States Government. Neither the United States Government nor any agency thereof, nor any of their employees, make any warranty, express or implied, or assumes any legal liability or responsibility for the accuracy, completeness, or usefulness of any information, apparatus, product, or process disclosed, or represents that its use would not infringe privately owned rights. Reference herein to any specific commercial product, process, or service by trade name, trademark, manufacturer, or otherwise does not necessarily constitute or imply its endorsement, recommendation, or favoring by the United States Government or any agency thereof. The views and opinions of authors expressed herein do not necessarily state or reflect those of the United States Government or any agency thereof. 


\section{DISCLAMMER}

Portions of this document may be illegible in electronic image products. Images are produced from the best available original document. 


\section{INTRODUCTION}

In a previous paper (I), we examined classical and semiclassical relativistic orbits of a charged particle of mass $m$ and charge $q$ on the surface of a rotating sphere threaded by an intense magnetic dipole field [1]. In that treatment, the dipole and rotation axes were taken as parallel for simplicity. The present note lifts that physically unrealistic assumption and treats the case of arbitrary relative tilt of the two axes.

The distinctive physical regimes are characterised by comparison of three dimensionless parameters: $\beta_{0}=|q| B_{0} R /\left(2 m c^{2}\right)$ is the magnetic field strength $B_{0}$ in rescaled units at the magnetic poles; $\epsilon=E /\left(m c^{2}\right)$ is the energy $E$ in units of the rest mass; and $l_{\phi}=2 c P_{\phi} /\left(q B_{0} R^{2}\right)$ is the canonical azimuthal angular momentum $P_{\phi}$ in rescaled units about the rotation axis. For convenience, we also define $l_{\theta}=2 c P_{\theta} /\left(q B_{0} R^{2}\right)$, where $P_{\theta}$ is the polar angular momentum.

For electrons on a typical neutron star, $\beta_{0} \simeq(0.1) R / 2 \lambda_{C} \sim 2.5 \times 10^{15}$, where $\lambda_{C}=$ electron Compton wavelength $=2 \pi \hbar / m_{e} c$ [2]. The relevant energy is then not set by $m c^{2}$, but by $\eta \equiv \epsilon / \beta_{0}$. The regime of $\eta \gtrsim 1$ is the ultrahigh energy regime, for $E \simeq 10^{21} \mathrm{eV}$ or higher. The magnetic field $\beta_{0}$ also sets the scale for $P_{\phi}$ : when $\left|l_{\phi}\right| \sim 1$, the ultrahigh $P_{\phi}$ regime, the effect of $P_{\phi}$ can overcome the binding effect of the field. Note that $P_{\phi}$ is the canonical, not the kinetic, azimuthal angular momentum. When both $\eta$ and $l_{\phi}$ are small, the charged particle has no allowed region on the spherical surface except very close to the rotational and magnetic poles. A large $l_{\phi}$ allows narrow regions away from the poles, but only $\eta \gtrsim 1$ allows charged particle motion over a substantial portion of the sphere.

We continue to use the metric signature (+- - ) with $x^{\mu}=(\mathbf{x}, c t)$, retaining $c$ and $\hbar$ explicitly. Equations from the paper I are referred to by equation number as (I.x) for

equation $(\mathrm{x})$. The rotational angular velocity is rescaled to $\bar{\omega}=\Omega R / c$, which is $\lesssim 0.1$ in realistic cases [2]. We take $\bar{\omega}=0.1$ throughout as illustrative. 


\section{MAGNETIC FIELD, CO-ORDINATES, AND METRIC}

We choose our axes so that the magnetic dipole is along the $\theta=0$ direction. The threading dipole magnetic field has polar strength $B_{0}$ :

$$
\begin{aligned}
& A_{\theta}=0 \\
& A_{r}=0 \\
& A_{\phi}=\left(B_{0} R^{2} / 2\right) \sin ^{2} \theta,
\end{aligned}
$$

where $A_{\phi}$ is defined in the rotating spherical co-ordinates.

The rotation axis is tilted at an angle $\theta_{0}$ with respect to the magnetic dipole in the $\phi=$

$0, \pi$ plane (Figure 1 ). (This apparently strange choice of co-ordinates is motivated by the the fact that the magnetic still dominate the rotational effects.) The metric in a spherical polar co-ordinate system $(r, \theta, \phi)$ rotating with the sphere is given by the line element

$$
\begin{aligned}
d s^{2} & =g_{\mu \nu} d x^{\mu} d x^{\nu} \\
& =c^{2}\left(1-\omega^{2}\right) d t^{2}-r^{2}\left(d \theta^{2}+\sin ^{2} \theta d \phi^{2}\right)-2 c r \omega_{\phi} \sin \theta d t d \phi-2 c r \omega_{\theta} d t d \theta .
\end{aligned}
$$

The vector $\boldsymbol{\omega}$ is defined from the rotational angular velocity vector $\Omega$ by $\omega=\Omega \times r / c$. Its components are

$$
\begin{aligned}
\omega_{\phi} & =\bar{\omega}\left[\cos \theta_{0} \sin \theta-\sin \theta_{0} \cos \theta \cos \phi\right], \\
\omega_{\theta} & =-\bar{\omega} \sin \theta_{0} \sin \phi, \\
\omega^{2} & =\omega_{\phi}^{2}+\omega_{\theta}^{2} .
\end{aligned}
$$

These are the appropriate generalisations of refs. $[1,3]$ to the case $\theta_{0} \neq 0$.

The Lagrangian of paper I is expressed in terms of the proper time $\tau$, with $\dot{x}^{\mu} \equiv d x^{\mu} / d \tau$. The equations (I.7) are three equations, one for each momentum component, suppressing radial motion. Since the Lagrangian does not explicitly depend on $t$, we have

$$
\frac{\partial L}{\partial t}=0 \quad \text {. }
$$


which implies

$$
\frac{d P_{0}}{d \tau}=0
$$

Because

$$
d \tau=\frac{d t \sqrt{g_{\mu \nu}\left(d x^{\mu} / d t\right)\left(d x^{\nu} / d t\right)}}{c}
$$

we have also

$$
\frac{d P_{0}}{d t}=0
$$

The equations of motion for $\phi$ and $\theta$ are non-trivial:

$$
\frac{\partial L}{\partial \phi}-\frac{d P_{\phi}}{d \tau}=0
$$

and

$$
\frac{\partial L}{\partial \theta}-\frac{d P_{\theta}}{d \tau}=0
$$

The constraint (I.6) is

$$
g^{00} P_{0}^{2}+2 g^{0 i} P_{0}\left(P_{i}-\frac{q}{c} A_{i}\right)+g^{i j}\left(P_{i}-\frac{q}{c} A_{i}\right)\left(P_{j}-\frac{q}{c} A_{j}\right)=(m c)^{2}
$$

but now includes a $g^{0 \theta}$ term in addition to the $g^{0 \phi}$ term. The contravariant metric components are

$$
\begin{gathered}
g^{00}=1 / c^{2}, \quad g^{r r}=-1 / r^{2}, \\
g^{\theta \theta}=-\left(1-\omega_{\theta}^{2}\right) / r^{2}, \quad g^{\phi \phi}=-\left(1-\omega_{\phi}^{2}\right) /\left(r^{2} \sin ^{2} \theta\right), \\
g^{0 \phi}=-\omega_{\phi} /(c r \sin \theta), \quad g^{0 \theta}=-\omega_{\theta} /(c r),
\end{gathered}
$$

where $r=R$ for our case. Along the actual worldpath in spacetime, the constraint $g_{\mu \nu} \dot{x}^{\mu} \dot{x}^{\nu}$ $=c^{2}$ obtains; this condition is valid after varying the action and simplifies the equations of motion. 


\section{APPROXIMATE ORBITS}

\section{A. Adiabatic Quasi-invariance}

As in paper I, the energy $E=P_{0}$ is exactly conserved, but $P_{\phi}$ or $l_{\phi}$ no longer is, unless $\sin \theta_{0}$ or $\bar{\omega}$ is zero. $P_{\theta}$ or $l_{\theta}$ was never conserved, but in paper I, its motion was exactly separable with $l_{\phi}$ conserved. That separability is also lost if $\bar{\omega} \sin \theta_{0} \neq 0$.

When $\theta_{0} \neq 0$, the non-conservation of $l_{\phi}$ is apparent from the equation of motion (10):

$$
\frac{d l_{\phi}}{d \tau}=\frac{\operatorname{sgn}(q)}{c R \beta_{0}}\left[\frac{1}{2} \frac{\partial \omega^{2}}{\partial \phi}+\frac{\partial \omega_{\phi}}{\partial \phi}(\operatorname{cr} \dot{\phi} \dot{\sin } \theta)+\frac{\partial \omega_{\theta}}{\partial \phi}(\operatorname{cr} \dot{\theta} \dot{t})\right],
$$

using the new metric for $\theta_{0} \neq 0$. We see that the non-conservation of $l_{\phi}$ is also $\mathcal{O}\left(1 / \beta_{0}\right)$, as well as requiring $\bar{\omega} \sin \theta_{0} \neq 0$. So in the limit of ultra-intense magnetic field $\beta_{0}$, the motion is not qualitatively different from the zero-tilt case. The orbits are more involved and possibly chaotic, but so focussed by the intense field that to zeroth order in $\left(1 / \beta_{0}\right)$, the results of paper I with $\theta_{0}=0$ are still qualitatively valid (Figure 4).

The approximate conservation of $l_{\phi}$ serves as the basis for a classical perturbative treatment of the particle orbits. In paper I, where $l_{\phi}$ was exactly conserved, the action variable

$$
J_{\phi}=\oint d \phi P_{\phi}
$$

was an adiabatic invariant $[3,4]$. It was this fact that allowed semiclassical quantisation of $P_{\phi}$ as a trivial step and the exactly separable treatment of $P_{\theta}$ as well.

\section{B. Classical Motion}

We thus expect any effects of the non-zero tilt to be suppressed by powers of $\bar{\omega}$. Since $\bar{\omega} \lesssim 0.1$, a combined perturbative treatment in powers of $\bar{\omega}$ and $1 / \beta_{0}$ is valid.

Since the $\phi$ motion to zeroth order in $1 / \beta_{0}$ is periodic, global expressions involving the $\phi$ co-ordinate (such as the energy, but not the orbit $\phi(\tau), \theta(\tau)$ ) can be averaged over the full circle $\phi \in[0,2 \pi]$ and $l_{\phi}$ treated as constant, in order to determine the first-order corrections [4]. The $\mathcal{O}\left(1 / \beta_{0}\right)$ correction to $d l_{\phi} / d \tau=0$ is obtained from averaging (14) over $\phi$ : 


$$
\left\langle\frac{d l_{\phi}}{d \tau}\right\rangle_{\phi}=\frac{\operatorname{sgn}(q)}{m c R \beta_{0}}\left\langle\frac{\partial L}{\partial \phi}\right\rangle_{\phi}=0
$$

where all the terms are $\propto \cos \phi$, $\sin \phi$, or $\cos \phi \sin \phi$ and average to zero. Consequently, the non-conservation of $l_{\phi}$, on average, actually starts at $\mathcal{O}\left(1 / \beta_{0}^{2}\right)$.

We consider the same set of limiting cases as in paper I. In the limit of ultrahigh energy, i.e, $\beta_{0} \gg l_{\phi}$, the constraint (8) with equations (9) and (10) becomes:

$$
l_{\theta} / \beta_{0}=\frac{2 \omega_{\theta} \pm \sqrt{\left(2 \omega_{\theta}\right)^{2}+4\left(1-\omega_{\theta}^{2}\right)\left[\eta^{2}+2 \eta \omega_{\phi} \sin \theta-\left(1-\omega_{\phi}^{2}\right) \sin ^{2} \theta\right]}}{2\left(1-\omega_{\theta}^{2}\right)},
$$

retaining all powers of $\eta$. Figures 2 and 3 show the allowed regions for several combinations of energy and tilt angle, where the allowed regions are defined by the requirement that $l_{\theta}$ in (13) be real. Note that the allowed regions of Figures 2 and 3 are no longer functions of $\theta$ alone, unlike the case of paper I, equations (I.24-25). The axial symmetry about the dipole is lost, but for moderate values of $\bar{\omega}$, the asymmetry is not extreme. For large tilts $\left(\theta_{0} \rightarrow \pi\right)$, the energy $\eta$ must be somewhat higher than in the zero-tilt case to make the entire sphere allowed. If $\phi$ averaging is applied to the constraint (17), the axial symmetry about the dipole is restored.

The limit where $l_{\phi}$ cannot be neglected, but $\eta \ll l_{\phi}$, is the ultrahigh $P_{\phi}$ case; $l_{\theta}$ is then zero except within the narrow allowed regions. If the tilt is zero (I.26-29), the particle orbits are almost infinitely narrow circles at constant $\theta$. The $\theta$ motion is $\mathcal{O}\left(1 / \beta_{0}\right)$ relative to the $\phi$ motion. With non-zero tilt and $l_{\phi}$ approximately conserved, the quasi-circular orbits occur at the same angle as in paper $\mathrm{I}, \sin \bar{\theta}=\sqrt{l_{\phi}}$, for $l_{\phi} \geq 0$, with $\mathcal{O}\left(1 / \beta_{0}\right)$ and $\mathcal{O}\left(\bar{\omega} \sin \theta_{0} / \beta_{0}\right)$ corrections.

The same argument holds for the small angle case, which is related to the ultrahigh $P_{\phi}$ limit in the limit $l_{\phi} \rightarrow 0$, with $\eta$ still negligible. The approximate conservation of $l_{\phi}$ still goes through in the limit of large $\beta_{0}$, with additional corrections of relative order $\bar{\omega} \sin \theta_{0}$.

The magnetic flux enclosed by an orbit is, to leading order in $1 / \beta_{0}$, just the same as in paper I, equations (I.32,36-37). The averaged rotational corrections are of order $\mathcal{O}\left(\bar{\omega}^{2}\right)$. 


\section{Semiclassical Quantization}

In the present case, the quantisation of $l_{\phi}$ in the Wilson-Sommerfeld conditions (I.33-34) is only approximate, but valid through $\mathcal{O}\left(1 / \beta_{0}\right)$.

The ultrahigh energy case is not qualitatively different if the tilt is non-zero. As we see from the classical motion, some regions of the sphere are forbidden for large tilt and moderate $\eta$. The quantum energy levels are still approximately the harmonic oscillator levels (I.39), depending only weakly on the field.

The ultrahigh $P_{\phi}$ case results in the energy levels:

$$
\begin{aligned}
E \simeq m c^{2}\left\{1+\left[1-\left(5 \bar{\omega}^{2} / 4\right) \sin ^{2} \theta_{0}+\bar{\omega}^{2} l_{\phi} \cos ^{2} \theta_{0}\right]\left(n_{\theta}+1 / 2\right) \hbar \beta_{0} /\left(2 m c R \sqrt{l_{\phi}}\right)\right. \\
\left.-\left(\bar{\omega}^{2} / 2\right)\left(\sin ^{2} \theta_{0}+l_{\phi} \cos ^{2} \theta_{0}\right)\right\}^{1 / 2}
\end{aligned}
$$

to leading order in $\beta_{0}$ and $\bar{\omega}$, with non-zero tilt. Note that the rotational corrections enter at $\mathcal{O}\left(\bar{\omega}^{2}\right)$. The corrections multiplying $\beta_{0}$ can either raise or lower the energy, but the last correction (the centrifugal effect) always lowers the energy.

The localised pole states are also corrected by rotational effects. Their levels are:

$$
E \simeq m c^{2}\left\{1+\left[1-\left(3 \bar{\omega}^{2} / 4\right) \sin ^{2} \theta_{0}\right]\left(n_{\theta}+1 / 2\right) \hbar \beta_{0} /(2 m c R)-\left(\bar{\omega}^{2} / 4\right) \sin ^{2} \theta_{0}\right\}^{1 / 2}
$$

which reproduce (I.35) if $\bar{\omega} \rightarrow 0$. For both this and the ultrahigh $P_{\phi}$ cases, the rotational corrections begin at $\mathcal{O}\left(\bar{\omega}^{2}\right)$ because of the angular averaging. Corrections of $\mathcal{O}(\bar{\omega})$ may enter, but suppressed by relative higher powers of $1 / \beta_{0}$.

\section{SUMMARY}

The physical situation of paper I [1] has been reconsidered with non-zero tilt $\theta_{0}$ between the rotational and magnetic dipole axes. For moderate rotational velocities $\bar{\omega}=\Omega R / c \lesssim$ 0.1 , the $\phi$-averaged corrections are $\mathcal{O}\left(\bar{\omega}^{2}\right)$. Corrections not explicitly treated are $\mathcal{O}\left(1 / \beta_{0}\right)$, $\mathcal{O}\left(\bar{\omega} / \beta_{0}\right)$, and $\mathcal{O}\left(\bar{\omega} \sin \theta_{0} / \beta_{0}\right)$. 
The multiplicative rotational corrections to the magnetic field in the energy arise from the effective electric field induced, as seen by an outside inertial observer, by the time-dependent, rotating magnetic field. Purely rotational (centrifugal) corrections also enter.

\section{ACKNOWLEDGMENTS}

We thank James M. Gelb (Univ. Texas at Arlington) for helpful suggestions. This work was supported by the Institute for Fundamental Theory and the U.S. Department of Energy Contract No. DE-FG05-86-ER40272 (Univ. Florida). 


\section{REFERENCES}

*E-mail: gopi@phys.ufl.edu.

† E-mail: kennedy@phys.uf.edu.

[1] K. S. Gopinath, D. C. Kennedy, and J. M. Gelb, Univ. Florida preprint UF-IFT-HEP97-2 and LANL preprint archive (http://xxx.lanl.gov/) astro-ph \# 9702014.

[2] S. L. Shapiro and S. A. Teukolsky, Black Holes, White Dwarfs, and Neutron Stars: The Physics of Compact Objects (John Wiley \& Sons, New York, 1983). F. C. Michel, Theory of Neutron Star Magnetospheres (University of Chicago Press, Chicago, 1991). M. S. Longair, High Energy Astrophysics, 2nd ed., 2 vols. (Cambridge University Press, Cambridge, 1992).

[3] L. D. Landau and E. M. Lifshitz, Classical Theory of Fields, translated by M. Hamermesh (Butterworth-Heinemann, Oxford, 1975).

[4] L. D. Landau and E. M. Lifshitz, Mechanics, 3rd ed., translated by J. B. Sykes and J. S. Bell (Pergamon, Oxford, 1976). 


\section{FIGURES}

FIG. 1. The sphere of radius $R$ threaded by field of magnetic dipole $\mathbf{M}$ and rotating about non-parallel axis with angular velocity $\Omega$ tilted at $\theta_{0}$ with respect to dipole.

FIG. 2. Allowed regions of real $l_{\theta}$ in the ultrahigh energy limit, for $\eta=0.5$. Surface co-ordinates are colatitude $\theta$ and longitude $\phi$. (a) $\theta_{0}=45^{\circ}$. (b) $\theta_{0}=135^{\circ}$.

FIG. 3. Allowed regions of real $l_{\theta}$ in the ultrahigh energy limit, for $\eta=1.0$. Surface co-ordinates are colatitude $\theta$ and longitude $\phi$. (a) $\theta_{0}=45^{\circ}$. (b) $\theta_{0}=135^{\circ}$.

FIG. 4. Phase space for in the ultrahigh $P_{\phi}$ case, with negligible $\eta$. Effects of $\mathcal{O}\left(1 / \beta_{0}\right)$ are greatly exaggerated. (a) Phase space for $\phi$, showing small variation of $l_{\phi}$ in case of $\theta_{0} \neq 0$. Allowed region (phase space trajectory) itself has finite thickness $\mathcal{O}\left(1 / \beta_{0}\right)$ (not shown). (b) Phase space for $\theta$, showing small variation of $\theta$ and small values of $l_{\theta}$. 


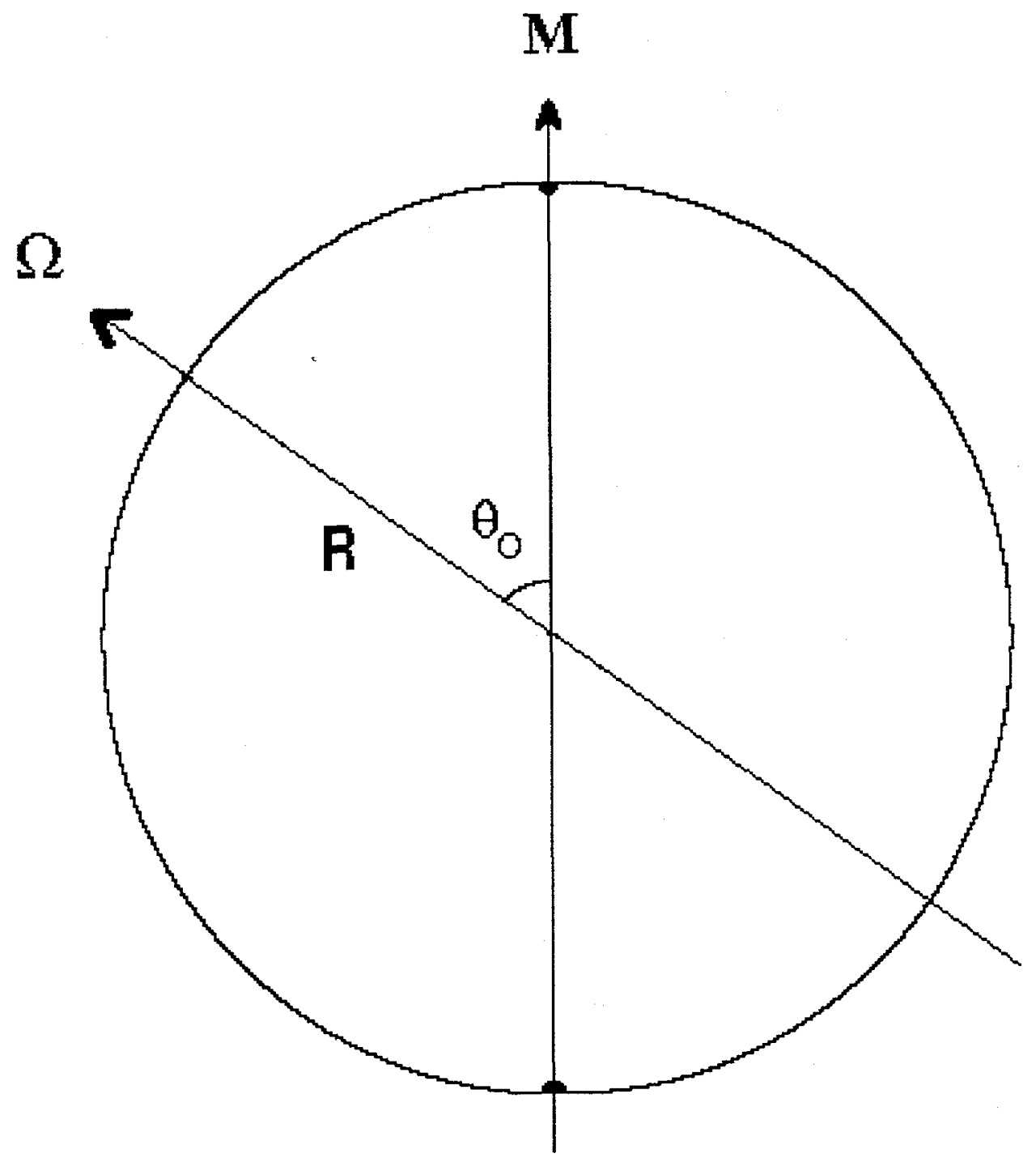

Figure 1 


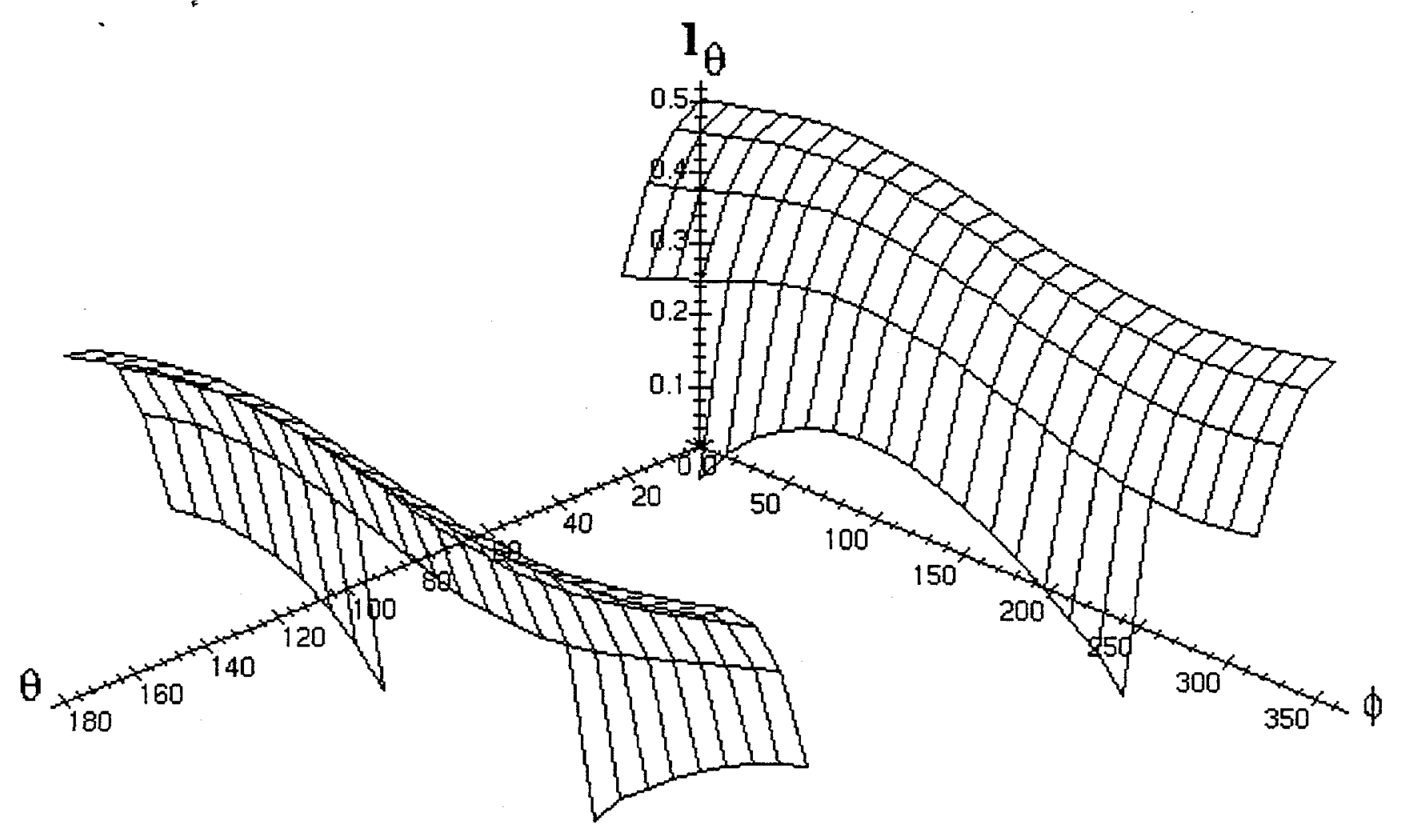

(a)

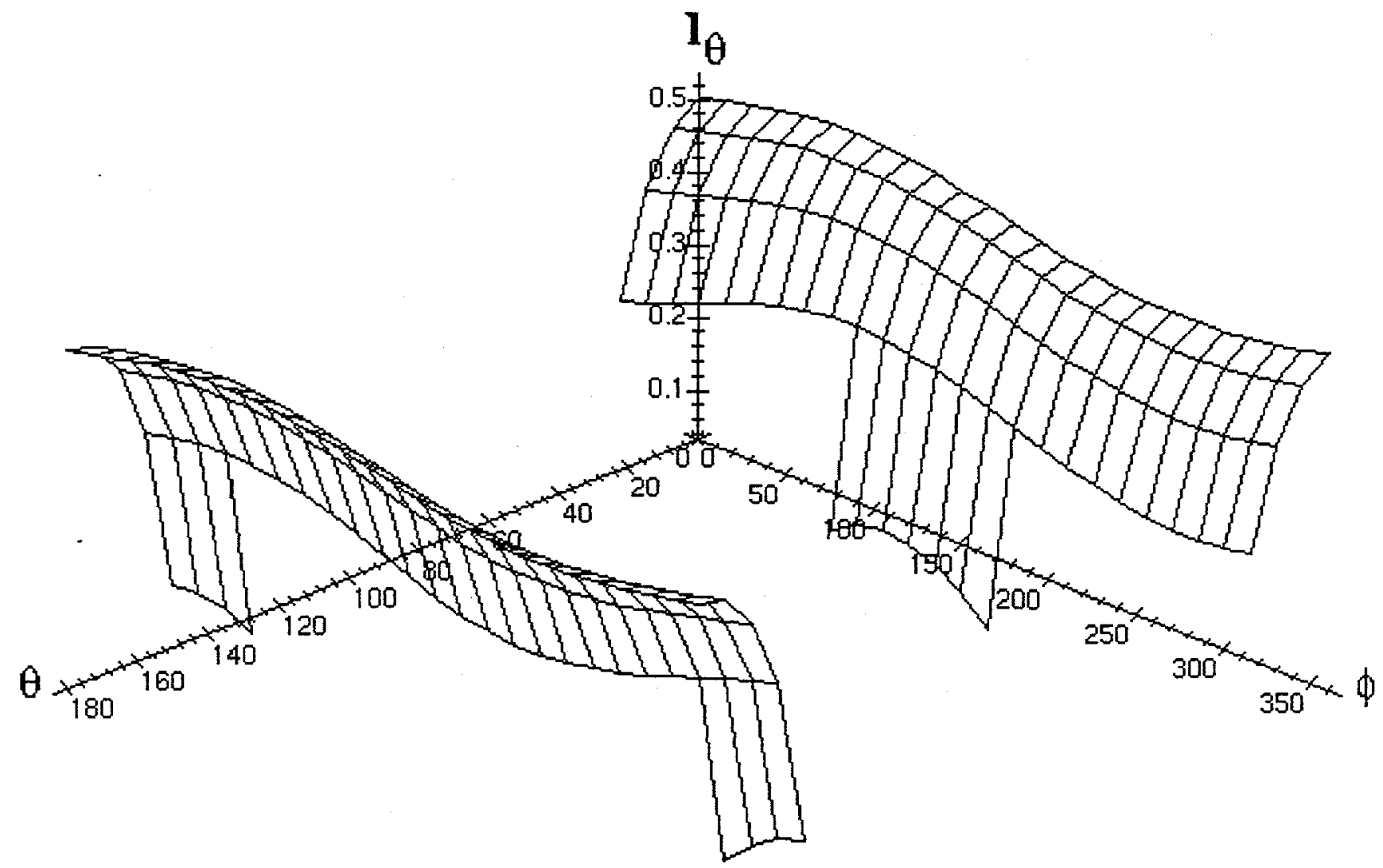

(b)

Figure 2 


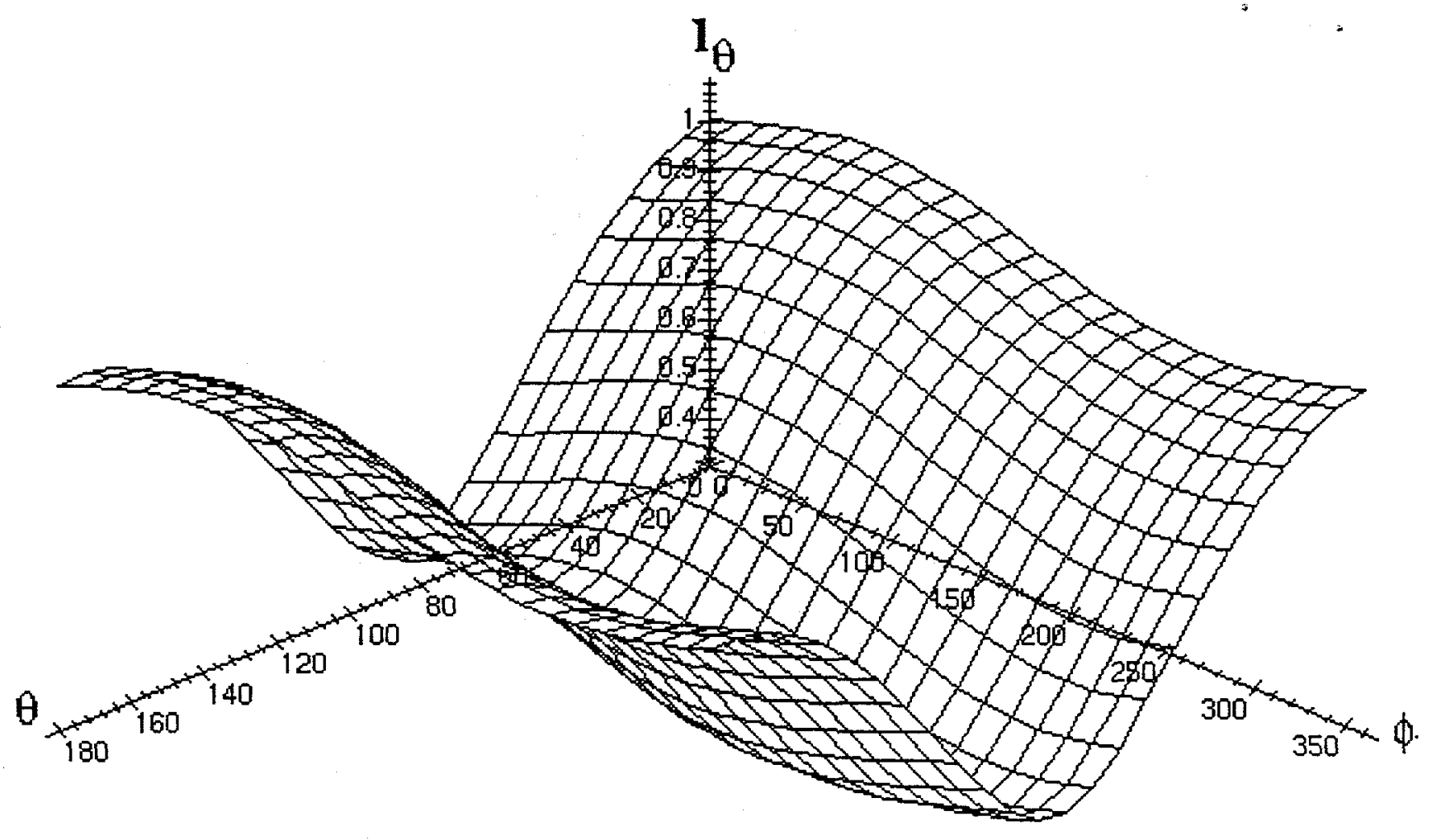

(a)

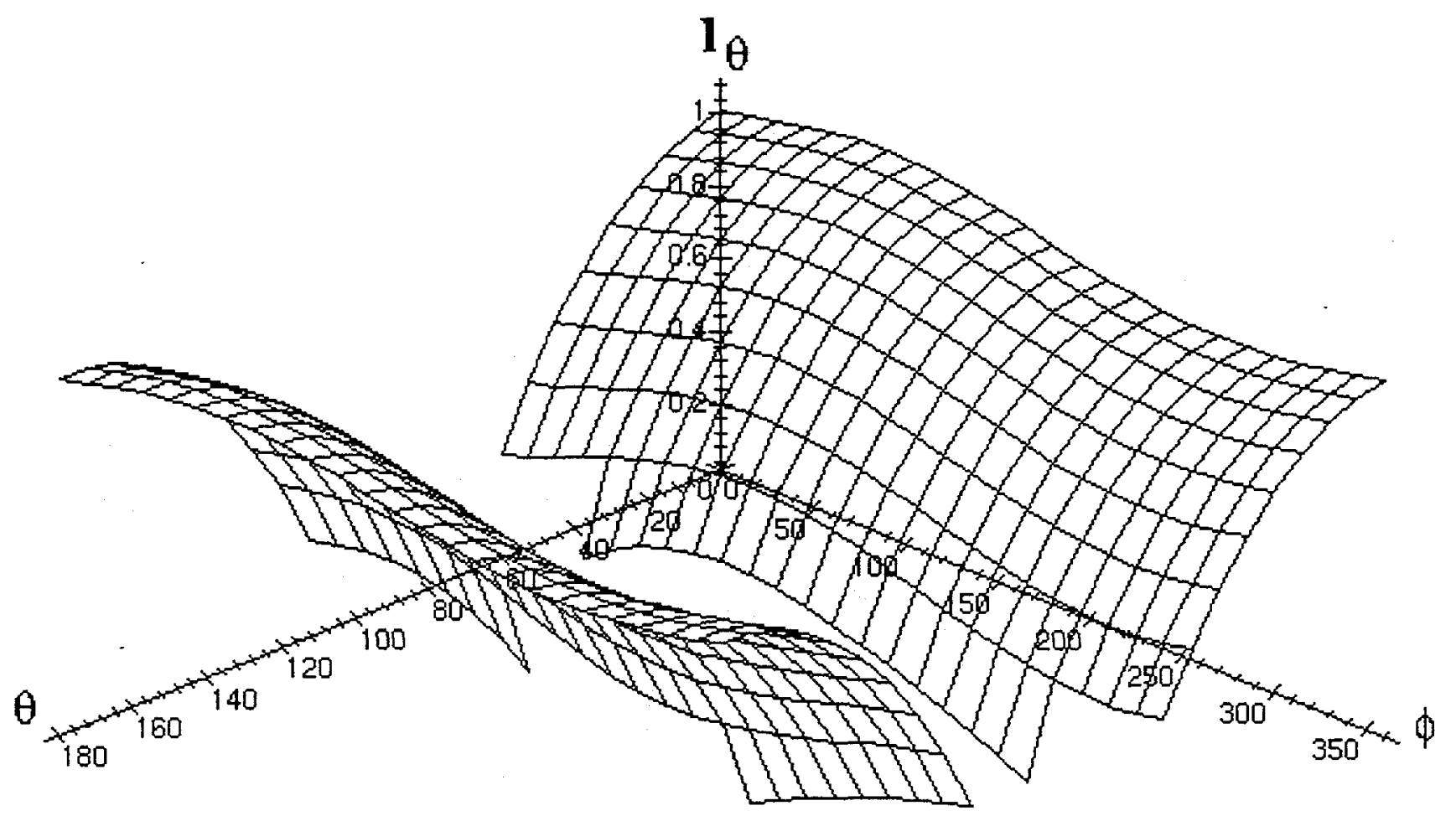

(b)

Figure 3 


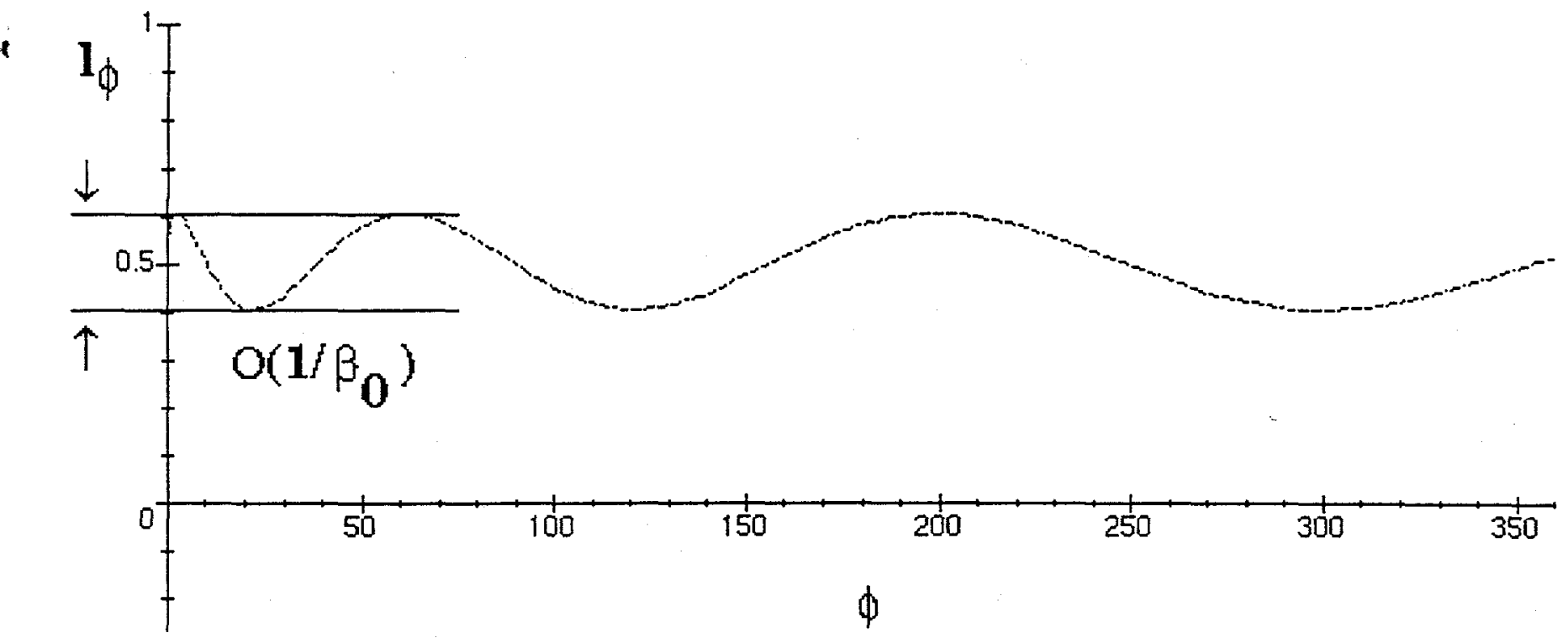

(a)

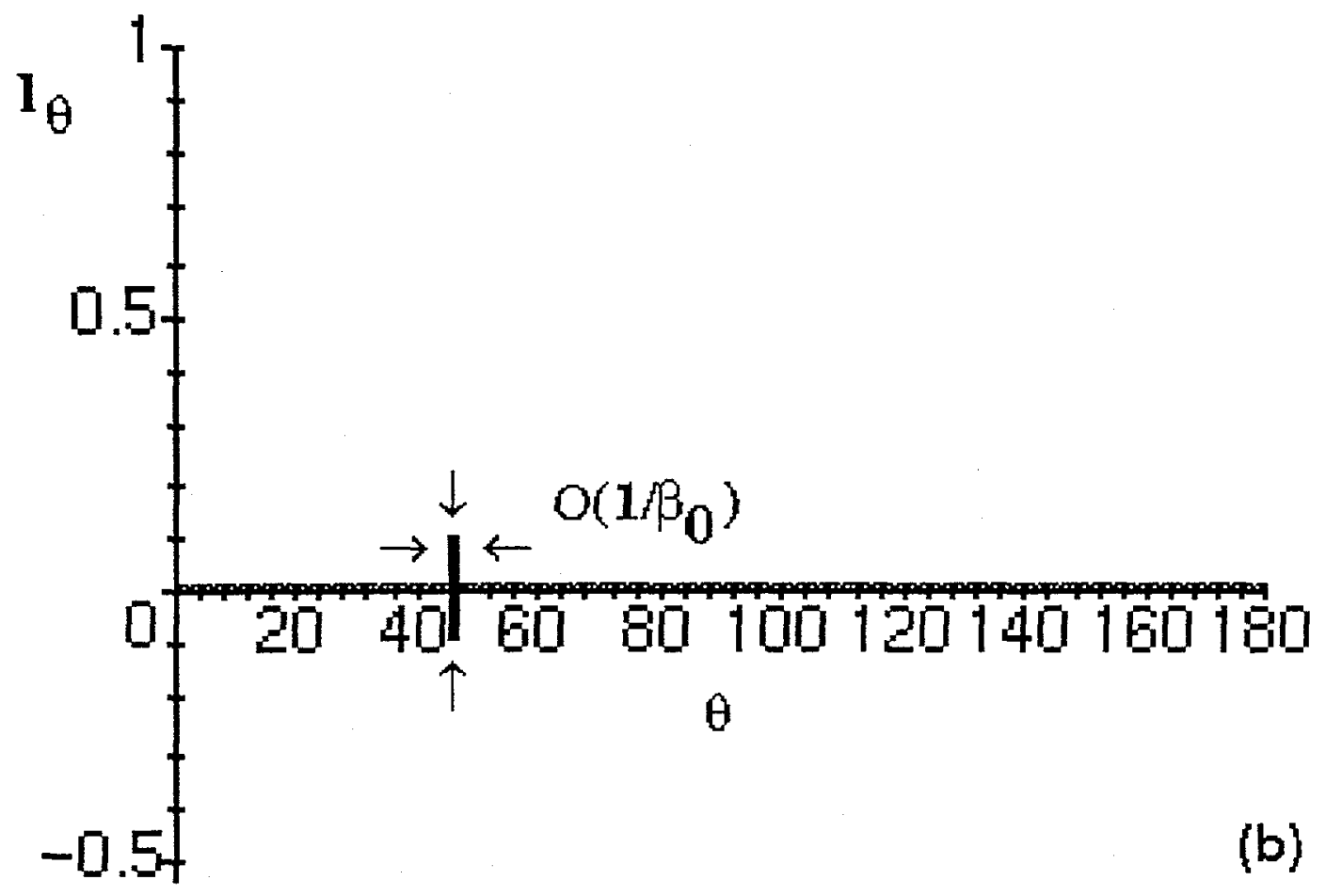

Figure 4 